\title{
A Proximal Analytic Center Cutting Plane Algorithm for Solving Variational Inequality Problems
}

\author{
Jie Shen ${ }^{1}$ and Li-Ping Pang ${ }^{2}$ \\ ${ }^{1}$ School of Mathematics, Liaoning Normal University, Dalian 116029, China \\ 2 School of Mathematical Sciences, Dalian University of Technology, \\ Dalian 116024, China
}

Correspondence should be addressed to Jie Shen, tt010725@163.com

Received 26 July 2012; Accepted 19 December 2012

Academic Editor: Jen Chih Yao

Copyright (C) 2012 J. Shen and L.-P. Pang. This is an open access article distributed under the Creative Commons Attribution License, which permits unrestricted use, distribution, and reproduction in any medium, provided the original work is properly cited.

\begin{abstract}
Under the condition that the values of mapping $F$ are evaluated approximately, we propose a proximal analytic center cutting plane algorithm for solving variational inequalities. It can be considered as an approximation of the earlier cutting plane method, and the conditions we impose on the corresponding mappings are more relaxed. The convergence analysis for the proposed algorithm is also given at the end of this paper.
\end{abstract}

\section{Introduction}

According to [1], the history of algorithms for solving finite-dimensional variational inequalities is relatively short. A recent development of such methods is the analytic center method based on cutting plane methods. It combines the feature of the newly developed interior point methods with the classical cutting plane scheme to achieve polynomial complexity in theory and quick convergence in practice. More details can be found in [2,3]. Specifically, Goffin et al. [4] developed a convergent framework for finding a solution $x^{*}$ of the variational inequality $\operatorname{VI}_{P}(F, X)$ associated with the continuous mapping $F$ from $X$ to $R^{n}$ and the polyhedron $X=\left\{x \in R^{n} \mid A x \leq b\right\}$ under an assumption slightly stronger than pseudomonotonicity. Again, Marcotte and Zhu [5] extended this algorithm to quasimonotone variational inequalities that satisfy a weak additional assumption. Such methods are effective in practice. 
Note that the facts in optimization problems, see [6-8], some functions from $R^{n}$ to $R$ are themselves defined through other minimization problems. For example, consider the Lagrangian relaxation, see [9-12], the primal problem is

$$
\max \{q(\xi) \mid \xi \in P, h(\xi)=0\},
$$

where $P$ is a compact subset of $R^{m}$ and $q: R^{m} \rightarrow R, h: R^{m} \rightarrow R^{n}$ are two functions. Lagrangian relaxation in this problem leads to the problem $\min \left\{f(x) \mid x \in R^{n}\right\}$, where

$$
f(x)=\max _{\xi \in P}\{q(\xi)+\langle x, h(\xi)\rangle\}
$$

is the dual function. Trying to solve problem (1.1) by means of solving its dual problem $\min \left\{f(x) \mid x \in R^{n}\right\}$ becomes more difficult since in this case evaluating the function value $f(x)$ requires solving exactly another optimization problem (1.2). Let us see another example: consider the problem

$$
\min \{f(x) \mid x \in C\}
$$

where $f$ is convex (not necessarily differentiable), $C \subset R^{n}$ is a nonempty closed convex set, $F$ is called the Moreau-Yosida regularization of $f$ on $C$, that is,

$$
F(x)=\min _{z \in C}\left\{f(z)+\frac{1}{2 \alpha}\|z-x\|^{2}\right\}
$$

where $\alpha$ is a positive parameter. A point $x \in C$ is a solution to (1.3) if and only if it is a solution to the problem:

$$
\min _{x \in R^{n}} F(x)
$$

The problem (1.5) is easier to deal with than (1.3), see [13]. But in this case, computing the exact function value of $F$ at an arbitrary point $x$ is difficult or even impossible since $F$ itself is defined through a minimization problem involving another function $f$. Intuitively, we consider the approximate computation of function $F$.

The above-mentioned phenomenon also exists for mappings from $X$ to $Y$, where $X$ and $Y$ are two subspaces of any two finite-dimensional spaces, respectively. Once a mapping, and more specifically, a continuous mapping is defined implicitly rather than explicitly, the approximation of the mapping becomes inevitable, see [14]. In this paper we try to solve $\mathrm{VI}_{P}(F, X)$ by assuming the values of the mapping $F$ from $X$ to $R^{n}$ can be only computed approximately. Under the assumption, we construct an algorithm for solving the approximate variational inequality problem $\operatorname{AVI}_{P}(F, X)$ and we also prove that there exists a cluster point of the iteration points generated by the proposed algorithm, it is a solution to the original problem $\operatorname{VI}_{P}(F, X)$.

This paper is organized as follows. Some basic concepts and results are introduced in Section 2. In Section 3, a proximal analytic center cutting plane algorithm for solving the variational inequality problems is given. The convergence analysis of the proposed algorithm is addressed in Section 4. In the last section, we give some conclusions. 


\section{Basic Concepts and Results}

Let $X=\left\{x \in R^{n} \mid A x \leq b\right\}$ be a polyhedron and $F$ a continuous mapping from $X$ to $R^{n}$. A vector $x^{*}$ is a solution to the variational inequality $\operatorname{VI}_{P}(F, X)$ if and only if it satisfies the system of nonlinear inequalities:

$$
\left\langle F\left(x^{*}\right), x-x^{*}\right\rangle \geq 0, \quad \forall x \in X
$$

The vector $x^{*}$ is a solution to the dual variational inequality $\operatorname{VI}_{D}(F, X)$ of $\operatorname{VI}_{P}(F, X)$ if and only if it satisfies

$$
\left\langle F(x), x-x^{*}\right\rangle \geq 0, \quad \forall x \in X
$$

We denote by $X_{P}^{*}$ the solution set of $\mathrm{VI}_{P}(F, X)$, and $X_{D}^{*}$ the solution set of $\mathrm{VI}_{D}(F, X)$, respectively. Whenever $F$ is continuous, we have $X_{D}^{*} \subseteq X_{P}^{*}$, see [15]. If $F$ is pseudomonotone on $X$, then $X_{D}^{*}=X_{P}^{*}$, see [15]. If $F$ is quasimonotone at $x^{*} \in X_{P}^{*}$ and $F\left(x^{*}\right)$ is not normal to $X$ at $x^{*}$, then $X_{D}^{*}$ is nonempty, see Proposition 1 in [5]. For the definitions of monotone, pseudomonotone and quasimonotone, see $[5,15]$.

Definition 2.1. The gap functions $g_{P}(x)$ and $g_{D}(x)$ of $\mathrm{VI}_{P}(F, X)$ and $\mathrm{VI}_{D}(F, X)$ are, respectively, defined by

$$
\begin{aligned}
& g_{P}(x)=\max _{y \in X}\langle F(x), x-y\rangle, \\
& g_{D}(x)=\max _{y \in X}\langle F(y), x-y\rangle .
\end{aligned}
$$

Note that $g_{P}(x) \geq 0, g_{D}(x) \geq 0$, and $g_{P}\left(x^{*}\right)=0$ if and only if $x^{*}$ is a solution to $\operatorname{VI}_{P}(F, X)$, $g_{D}\left(x^{*}\right)=0$ if and only if $x^{*}$ is a solution to $\mathrm{VI}_{D}(F, X)$. Thus, $X_{P}^{*}=\left\{x \in X \mid g_{P}(x)=0\right\}, X_{D}^{*}=$ $\left\{x \in X \mid g_{D}(x)=0\right\}$.

Definition 2.2. A point $\tilde{x} \in X$ is called an $\varepsilon$-solution to $\operatorname{VI}_{P}(F, X)$ if $g_{P}(\tilde{x}) \leq \varepsilon$ for given $\varepsilon>0$.

Definition 2.3. For $x, y \in X$, we say $F(x) \preceq F(y)$ if and only if $F_{i}(x) \leq F_{i}(y)$, for $i=1,2, \ldots, n$, where $F(x)=\left(F_{1}(x), F_{2}(x), \ldots, F_{n}(x)\right)^{T}$.

Assumptions 2.4. Throughout this paper, we make the following assumptions: for each $x, y \in$ $X$, given any $\bar{\varepsilon}=(\varepsilon, \varepsilon, \ldots, \varepsilon), \bar{\delta}=(\delta, \delta, \ldots, \delta)$, where $\varepsilon, \delta \in(0,1)$, we can always find a $\bar{F}_{x} \in R^{n}$ and a $\bar{F}_{y} \in R^{n}$ such that

(a) $F(x) \preceq \bar{F}_{x} \preceq F(x)+\bar{\varepsilon}, F(y) \preceq \bar{F}_{y} \preceq F(y)+\bar{\delta}$,

that is, we can compute the approximate value of $F$ with any precision;

(b) $\bar{F}_{y} \longrightarrow \bar{F}_{x}$ if $y \longrightarrow x$, no matter the relationship between $\bar{\varepsilon}$ and $\bar{\delta}$;

(c) $\left\|\bar{F}_{y}-\bar{F}_{x}\right\| \leq L\|y-x\|$, where $L>0$ is a constant. 
These assumptions are realistic in practice, see [16, 17]. By using the given architecture in $[16,17]$, we can approximate the mapping $F$ arbitrary well since neural networks are capable of approximating any function from one finite-dimensional real vector space to another one arbitrary well, see [18]. Specifically, let us consider the case of univariate function. If $f$ is a min-type function of the form

$$
f(x)=\inf \left\{N_{z}(x) \mid z \in Z\right\},
$$

where each $N_{z}(x)$ is convex and $Z$ is an infinite set, then it may be impossible to calculate $f(x)$. However, we may still consider two cases. In the first case of controllable accuracy, for each positive $\varepsilon>0$ one can find an $\varepsilon$-minimizer of (2.5), that is, an element $z_{x} \in Z$ satisfying $N_{z_{x}}(x) \leq f(x)+\varepsilon$; in the second case, this may be possible only for some fixed (any possibly unknown) $\varepsilon<\infty$. In both cases, we may set $\bar{f}_{x}=N_{z_{x}}(x) \leq f(x)+\varepsilon$. A special case of (2.5) arises from Lagrangian relaxation [15], where the problem $\max \{f(x) \mid x \in S\}$ with $S=R_{+}^{n}$ is the Lagrangian dual of the primal problem

$$
\inf \psi_{0}(x) \quad \text { s.t. } \psi_{j}(x) \geq 0, \quad j=1,2, \ldots, n, x \in X,
$$

with $N_{z}(x)=\psi_{0}(z)+\langle x, \psi(z)\rangle$ for $\psi=\left(\psi_{1}, \psi_{2}, \ldots, \psi_{n}\right)$. Then, for each multiplier $x \geq 0$, we need only to find $z_{x} \in Z$ such that $\bar{f}_{x}=N_{z_{x}}(x) \leq f(x)+\varepsilon$, see [19].

Under the above assumptions (2.4), we introduce an approximate problem $\operatorname{AVI}_{P}(F, X)$ associated with $\operatorname{VI}_{P}(F, X)$ : finding $x^{*} \in X$ such that

$$
\left\langle\bar{F}_{x^{*}}, x-x^{*}\right\rangle \geq 0, \quad \forall x \in X
$$

where $\bar{F}_{x^{*}}$ satisfies $F\left(x^{*}\right) \leq \bar{F}_{x^{*}} \leq F\left(x^{*}\right)+\bar{\varepsilon}$ for arbitrary $\bar{\varepsilon}>0$. Its dual problem $\operatorname{AVI}_{D}(F, X)$ is to find $x^{*} \in X$ such that

$$
\left\langle\bar{F}_{x}, x-x^{*}\right\rangle \geq 0, \quad \forall x \in X,
$$

where $\bar{F}_{x}$ satisfies $F(x) \preceq \bar{F}_{x} \preceq F(x)+\bar{\varepsilon}$ for arbitrary $\bar{\varepsilon}>0$.

Definition 2.5. The gap function of $\operatorname{AVI}_{P}(F, X)$ is defined by $\bar{g}_{P}(x)=\max _{y \in X}\left\langle\bar{F}_{x}, x-y\right\rangle$.

Definition 2.6. A point $\tilde{x} \in X$ is called an $\varepsilon$-solution to $\operatorname{AVI}_{P}(F, X)$ if $\bar{g}_{P}(\tilde{x}) \leq \varepsilon$ for given $\varepsilon>0$.

The optimal solution sets of $\operatorname{AVI}_{P}(F, X)$ and $\operatorname{AVI}_{D}(F, X)$ are denoted by $A X_{P}^{*}$ and $A X_{D^{\prime}}^{*}$ respectively. The following proposition ensures that $A X_{D}^{*}$ is nonempty.

Proposition 2.7. If there exists a point $x^{*} \in A X_{P}^{*}$ such that

$$
\left\langle\bar{F}_{x^{*}}, y-x^{*}\right\rangle>0 \Longrightarrow\left\langle\bar{F}_{y}, y-x^{*}\right\rangle \geq 0, \quad \forall y \in X
$$

and $\bar{F}_{x^{*}}$ is not normal to $X$ at $x^{*}$, then $A X_{D}^{*}$ is nonempty. 
Proof. Since $\bar{F}_{x^{*}}$ is not normal to $X$ at $x^{*}$, there exists a point $x_{0} \in X$ such that $\left\langle\bar{F}_{x^{*}}, x_{0}-x^{*}\right\rangle>0$. $\forall x \in X$, set $x_{t}=t x_{0}+(1-t) x$ for $t \in(0,1]$, then we have $\left\langle\bar{F}_{x^{*}}, x-x^{*}\right\rangle \geq 0$ and $\left\langle\bar{F}_{x^{*}}, x_{t}-x^{*}\right\rangle>0$. Note the condition (2.9), we obtain $\left\langle\bar{F}_{x_{t}}, x_{t}-x^{*}\right\rangle \geq 0$. Letting $t \rightarrow 0$, it follows from the condition (b) in (2.4) that $\left\langle\bar{F}_{x}, x-x^{*}\right\rangle \geq 0$, that is, $x^{*} \in A X_{D}^{*}$.

In the following part, we focus our attention on solving $\operatorname{AVI}_{P}(F, X)$. Let $\Gamma(y, x): R^{n} \times$ $R^{n} \rightarrow R^{n}$ denote an auxiliary mapping, continuous in $x$ and $y$, strongly monotone in $y$, that is,

$$
\langle\Gamma(y, x)-\Gamma(z, x), y-z\rangle \geq \beta\|y-z\|^{2}, \quad \forall y, z \in X
$$

for some $\beta>0$. We consider the auxiliary variational inequality associated with $\Gamma$, whose solution $w(x)$ satisfies

$$
\left\langle\Gamma(w(x), x)-\Gamma(x, x)+\bar{F}_{x}, y-w(x)\right\rangle \geq 0, \quad \forall y \in X
$$

In view of the strong monotonicity of $\Gamma(y, x)$ with respect to $y$, this auxiliary variational inequality has a unique solution $w(x)$.

Proposition 2.8. The mapping $w: X \rightarrow X$ is continuous on $X$. Furthermore, $x$ is a solution to $A V I_{P}(F, X)$ if and only if $x=w(x)$.

Proof. The first part of the proposition follows from Theorem 5.4 in [1]. To prove the second part, we first suppose that $x=w(x)$. This yields $\left\langle\bar{F}_{x}, y-x\right\rangle \geq 0, \forall y \in X$, that is, $x$ solves $\operatorname{AVI}_{P}(F, X)$. Conversely, suppose that $x$ solves $\operatorname{AVI}_{P}(F, X)$, then

$$
\left\langle\bar{F}_{x}, w(x)-x\right\rangle \geq 0
$$

and from (2.11), we have

$$
\left\langle\Gamma(w(x), x)-\Gamma(x, x)+\bar{F}_{x}, x-w(x)\right\rangle \geq 0
$$

Adding the two preceding inequalities, one obtains

$$
\langle\Gamma(w(x), x)-\Gamma(x, x), x-w(x)\rangle \geq 0
$$

and we conclude, from the strong monotonicity of $\Gamma$ with respect to $y$, that $x=w(x)$. satisfying

Let $\rho<1, \alpha<\beta$ be two positive numbers. Let $l$ be the smallest nonnegative integer

$$
\left\langle\bar{F}_{x+\rho^{l}(w(x)-x)}, x-w(x)\right\rangle \geq \alpha\|w(x)-x\|^{2},
$$


where $\bar{F}_{x+\rho^{l}(w(x)-x)}$ satisfies $F\left(x+\rho^{l}(w(x)-x)\right) \leq \bar{F}_{x+\rho^{l}(w(x)-x)} \preceq F\left(x+\rho^{l}(w(x)-x)\right)+\bar{\varepsilon}$ for arbitrary $\bar{\varepsilon}>0$. The existence of a finite $l$ will be proved in Proposition 2.9. The composite mapping $G$ is defined, for every $x \in X$, by

$$
G(x)=\bar{G}_{x}=\bar{F}_{x+\rho^{l}(w(x)-x)} .
$$

If $x^{*}$ is a solution to $\operatorname{AVI}_{P}(F, X)$, then we have $w\left(x^{*}\right)=x^{*}, l=0$ and $\bar{G}_{x^{*}}=\bar{F}_{x^{*}}$.

Proposition 2.9. The operator $G$ is well defined for every $x \in X$. Moreover, we have

$$
l \leq \frac{\ln ((\beta-\alpha) / L)}{\ln \rho}
$$

where $L$ is the number given in (2.4)-(c).

Proof. From the definition of $w(x)$, we have

$$
\left\langle\bar{F}_{x}, x-w(x)\right\rangle \geq\langle\Gamma(w(x), x)-\Gamma(x, x), w(x)-x\rangle \geq \beta\|x-w(x)\|^{2} .
$$

Suppose (2.15) does not hold for any finite integer $l$, that is,

$$
\left\langle\bar{F}_{x+\rho^{l}(w(x)-x)}, x-w(x)\right\rangle<\alpha\|x-w(x)\|^{2} .
$$

Note the assumption (2.4)-(b), we obtain

$$
\bar{F}_{x+\rho^{l}(w(x)-x)} \longrightarrow \bar{F}_{x} \quad \text { as } l \longrightarrow+\infty,
$$

therefore,

$$
\left\langle\bar{F}_{x}, x-w(x)\right\rangle \leq \alpha\|x-w(x)\|^{2}
$$

Since $\alpha<\beta,(2.21)$ is in contradiction with (2.18). To prove the second part, we notice that

$$
\begin{aligned}
\left\langle\bar{F}_{x+\rho^{l}(w(x)-x)}, x-w(x)\right\rangle & =\left\langle\bar{F}_{x}, x-w(x)\right\rangle+\left\langle\bar{F}_{x+\rho^{l}(w(x)-x)}-\bar{F}_{x}, x-w(x)\right\rangle \\
& \geq \beta\|w(x)-x\|^{2}-L \rho^{l}\|w(x)-x\|^{2} \\
& \geq \alpha\|w(x)-x\|^{2}
\end{aligned}
$$

if $\alpha \leq \beta-L \rho^{l}$, which means the second conclusion of Proposition 2.9 holds.

Proposition 2.10. If $x \notin A X_{P}^{*}$, then $\forall y^{*} \in A X_{D}^{*}$, we have

$$
\left\langle\bar{G}_{x}, x-y^{*}\right\rangle>0
$$


Proof. Let $y(x)=x+\rho^{l}(w(x)-x)$, then $\bar{G}_{x}=\bar{F}_{y(x)}$ and

$$
\left\langle\bar{F}_{y(x)}, w(x)-x\right\rangle \leq-\alpha\|w(x)-x\|^{2} .
$$

Since $x \notin A X_{P}^{*}$, so $w(x) \neq x$. Therefore,

$$
\left\langle\bar{F}_{y(x)}, y(x)-x\right\rangle=\rho^{l}\left\langle\bar{F}_{y(x)}, w(x)-x\right\rangle<0 .
$$

For all $y^{*} \in A X_{D^{*}}^{*}$, there holds

$$
\left\langle\bar{F}_{y(x)}, y(x)-y^{*}\right\rangle \geq 0
$$

By combining (2.25) with (2.26), we obtain $\left\langle\bar{F}_{y(x)}, x-y^{*}\right\rangle>0$, that is, $\left\langle\bar{G}_{x}, x-y^{*}\right\rangle>0$.

\section{A Proximal Analytic Center Cutting Plane Algorithm}

Algorithm 3.1 offered in this section is a modification of the algorithm in [5]. Algorithm 3.1 is described as follows.

Algorithm 3.1. Let $\beta$ be the strong monotonicity constant of $\Gamma(x, y)$ with respect to $y$, and let $\alpha \in(0, \beta), \varepsilon \in(0,1)$ be two constants. Set $k=0, A^{k}=A \in R^{m \times n}, b^{k}=b$, and $\varepsilon^{k}=\varepsilon$.

Step 1 (computation of the center). Find an approximate analytic center $x^{k}$ of $X^{k}=\left\{x \in R^{n} \mid\right.$ $\left.A^{k} x \leq b^{k}\right\}$.

Step 2 (stopping criterion). If $g_{P}\left(x^{k}\right) \leq \varepsilon$, stop.

Step 3 (solving the approximate auxiliary variational inequality problem). Find $w\left(x^{k}\right)$, such that

$$
\left\langle\bar{F}_{x^{k}}+\Gamma\left(w\left(x^{k}\right), x^{k}\right)-\Gamma\left(x^{k}, x^{k}\right), y-w\left(x^{k}\right)\right\rangle \geq 0, \quad \forall y \in X
$$

where $\bar{F}_{x^{k}}$ satisfies $F\left(x^{k}\right) \preceq \bar{F}_{x^{k}} \preceq F\left(x^{k}\right)+\bar{\varepsilon}_{x^{k}}, \bar{\varepsilon}_{x^{k}}=\left(\varepsilon^{k}, \varepsilon^{k}, \ldots, \varepsilon^{k}\right)^{T}$.

Step 4 (construction of the approximate cutting plane). Let $y^{k}=x^{k}+\rho^{l_{k}}\left(w\left(x^{k}\right)-x^{k}\right)$ and $\bar{G}_{x^{k}}=\bar{F}_{y^{k}}$, where $l_{k}$ is the smallest integer that satisfies

$$
\left\langle\bar{F}_{x^{k}+\rho^{k}\left(w\left(x^{k}\right)-x^{k}\right)}, x^{k}-w\left(x^{k}\right)\right\rangle \geq \alpha\left\|w\left(x^{k}\right)-x^{k}\right\|^{2}
$$

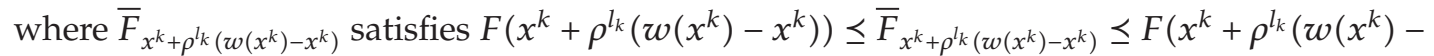
$\left.\left.x^{k}\right)\right)+\bar{\varepsilon}_{x^{k}+\rho^{l}\left(w\left(x^{k}\right)-x^{k}\right)}, \bar{\varepsilon}_{x^{k}+\rho^{l}\left(w\left(x^{k}\right)-x^{k}\right)}=\left(\varepsilon^{k}, \varepsilon^{k}, \ldots, \varepsilon^{k}\right)^{T}$. 
Let $H^{k}=\left\{x \mid\left\langle\bar{G}_{x^{k}}, x-x^{k}\right\rangle=0\right\}$,

$$
A^{k+1}=\left(\begin{array}{c}
A^{k} \\
\bar{G}_{x^{k}}
\end{array}\right), \quad b^{k+1}=\left(\begin{array}{c}
b^{k} \\
\bar{G}_{x^{k}}^{T} x^{k}
\end{array}\right) .
$$

Increase $k$ by one and go to Step 1 .

End of Algorithm 3.1

\section{Convergence Analysis}

In [20], the authors proposed a column generation scheme to generate the polytope $X^{k}$, and they proved if $k$ satisfies the following inequality

$$
\frac{\varepsilon^{2}}{m} \geq \frac{1 / 2+2 m \ln \left(1+(k+1) / 8 m^{2}\right)}{2 m+k+1} e^{-2 \alpha((k+1) /(2 m+k+1))},
$$

where $\varepsilon<1 / 2$ is a constant, the scheme will stop with a feasible solution, that is, they can find a vector $a_{k+1}$ such that $\left\{y \mid a_{k+1}^{T} y \leq a_{k+1}^{T} y^{k}\right\} \supset \Gamma$ with $\left\|a_{k+1}\right\|=1$, $\Gamma$ contains a full-dimensional closed ball with $\varepsilon<1 / 2$ radius. In other words, there exists the smallest $k(\varepsilon)$ such that $X^{k(\varepsilon)}$ generated by the column generation scheme does not contain the ball with $\varepsilon<1 / 2$ radius, and it is known as the finite cut property. It is easy to know that the result of Theorem 6.6 in [20] also holds without much change for our Algorithm 3.1 using the approximate centers. That is, by using the row generation scheme, there exists the smallest $k(\rho)$ such that $X^{k(\rho)}$ generated in Step 4 in Algorithm 3.1 does not contain the ball with $\rho$ radius lying inside the polytope $X$. This result plays an important role in proving the convergence of the described Algorithm 3.1 in Section 3.

Theorem 4.1. Let the polyhedron X have nonempty interior, and let $A X_{D}^{*}$ be nonempty. Assumption (2.4) holds. Then either Algorithm 3.1 stops with a solution to $A V I_{P}(F, X)$ after a finite number of iterations, or there exists a subsequence of the infinite sequence $\left\{x^{k}\right\}$ that converges to a point in $A X_{P}^{*}$.

Proof. Assume that $x^{k} \notin A X_{P}^{*}$ for every iteration $k$, and let $y^{*} \in A X_{D}^{*}$. From Proposition 2.10, we know that $y^{*} \in X^{k}$ never lies on $H^{k}$ for any $k$. Let $\left\{\bar{y}_{i}\right\}_{i \in N}$ be an arbitrary sequence of point in the interior of $X$ converging to $y^{*}$ and $\delta_{i}$ a sequence of positive numbers such that $\lim _{i \rightarrow+\infty} \delta_{i}=0$ and that the sequence of closed balls $\left\{B\left(\bar{y}_{i}, \delta_{i}\right)\right\}_{i \in N}$ lies in the interior of $X$. Note that $\lim _{i \rightarrow+\infty} B\left(\bar{y}_{i}, \delta_{i}\right)=\left\{y^{*}\right\}$. From finite cut property, we know that there exists the smallest index $k(i)$ and a point $\widetilde{y}_{i} \in B\left(\bar{y}_{i}, \delta_{i}\right)$ such that $\tilde{y}_{i}$ satisfies

$$
\left\langle\bar{G}_{x^{k(i)}}, x^{k(i)}-\tilde{y}_{i}\right\rangle<0
$$

As $\left\langle\bar{G}_{x^{k(i)}}, x^{k(i)}-y^{*}\right\rangle>0$, there exists a point $\hat{y}_{i}$ on the segment $\left[\tilde{y}_{i}, y^{*}\right]$ such that $\left\langle\bar{G}_{x^{k(i)}}, x^{k(i)}-\right.$ $\left.\hat{y}_{i}\right\rangle=0$. Since $X$ is compact, we can extract from $\left\{x^{k(i)}\right\}_{i \in N}$ a convergent subsequence $\left\{x^{k(i)}\right\}_{i \in S}$. Denote by $\hat{x}$ its limit point, we have

$$
\left\langle\bar{G}_{x^{k(i)}}, \widehat{y}_{i}-x^{k(i)}\right\rangle=0, \quad \forall i \in S .
$$


From Proposition 2.9, we know that $l_{k(i)}$ is bounded. Consequently, we can extract form $\left\{l_{k(i)}\right\}_{i \in S}$ a constant subsequence $l_{k^{*}(i)}=k^{*}$. Now from the continuity of $w(x)$ for fixed $k$ and the relations (2.15) and (4.3), it follows by taking the limit in (4.3) that

$$
\left\langle\bar{G}_{\widehat{x}}, y^{*}-\widehat{x}\right\rangle=0
$$

By Proposition 2.10, we conclude that $\widehat{x} \in A X_{P}^{*}$.

Theorem 4.2. Under the conditions of Theorem 4.1, either Algorithm 3.1 stops with a solution to $A V I_{P}(F, X)$ after a finite number of iterations, or there exists a subsequence of the infinite sequence $\left\{x^{k}\right\}$ that converges to a point in $X_{P}^{*}$.

Proof. Since $\varepsilon \in(0,1), \varepsilon^{k} \in(0,1)$. At the end of Step 4 in Algorithm 3.1 we increase $k$ by one, so we have $\varepsilon^{k+1}<\varepsilon^{k}, \varepsilon^{k} \rightarrow 0$ as $k \rightarrow \infty$. Moreover, $\bar{\varepsilon}_{x^{k}} \rightarrow \overline{0}$ as $k \rightarrow \infty$ in Algorithm 3.1, where $\overline{0}$ denotes the zero vector. This means $\bar{F}_{\widehat{x}}=F(\widehat{x})$ as $k \rightarrow \infty$. Therefore, from the second result of Theorem 4.1, we know $\hat{x}$ is the solution to the problem $\operatorname{VI}_{P}(F, X)$.

\section{Conclusions}

In [5], the authors proposed a cutting plane method for solving the quasimonotone variational inequalities, but throughout the paper they employed the exact information of the mapping $F$ from $X$ to $R^{n}$. Just like the discussion in the first part of our paper, sometimes, it is not so easy or even impossible to compute the exact values of the mapping $F$. Motivated by this fact, we consider constructing an approximate problem $\operatorname{AVI}_{P}(F, X)$ of $\mathrm{VI}_{P}(F, X)$, and try out a proximal analytic center cutting plane algorithm for solving $\operatorname{AVI}_{P}(F, X)$. In contrast to [5], our algorithm can be viewed as an approximation algorithm, and it is easier to implement than [5] since it only requires the inexact information of the corresponding mapping. At the same time, the conditions we impose on the corresponding mappings are more relaxed, for example, [5] requires the mapping $F$ satisfies the Lipschitz condition, but we only require that the so-called approximate Lipschitz condition (2.4)-(c) holds.

\section{Acknowledgments}

This work is partially supported by the National Natural Science Foundation of China (Grant nos. 11171138, 11171049) and Higher School Research Project of Educational Department of Liaoning Province (Grant no. L2010235).

\section{References}

[1] P. T. Harker and J.-S. Pang, "Finite-dimensional variational inequality and nonlinear complementarity problems: a survey of theory, algorithms and applications," Mathematical Programming B, vol. 48, no. 2, pp. 161-220, 1990.

[2] J.-L. Goffin and J.-P. Vial, "Convex nondifferentiable optimization: a survey focused on the analytic center cutting plane method," Optimization Methods E Software, vol. 17, no. 5, pp. 805-867, 2002.

[3] Z. Q. Luo and J. Sun, "An analytic center based column generation algorithm for convex quadratic feasibility problems," SIAM Journal on Optimization, vol. 9, no. 1, pp. 217-235, 1999.

[4] J. L. Goffin, P. Marcotte, and D. Zhu, "An analytic center cutting plane method for pseudomonotone variational inequalities," Operations Research Letters, vol. 20, no. 1, pp. 1-6, 1997. 
[5] P. Marcotte and D. L. Zhu, "A cutting plane method for solving quasimonotone variational inequalities," Computational Optimization and Applications, vol. 20, no. 3, pp. 317-324, 2001.

[6] M. Hintermüller, "A proximal bundle method based on approximate subgradients," Computational Optimization and Applications, vol. 20, no. 3, pp. 245-266, 2001.

[7] K. C. Kiwiel, "An algorithm for nonsmooth convex minimization with errors," Mathematics of Computation, vol. 45, no. 171, pp. 173-180, 1985.

[8] M. V. Solodov, "On approximations with finite precision in bundle methods for nonsmooth optimization," Journal of Optimization Theory and Applications, vol. 119, no. 1, pp. 151-165, 2003.

[9] D. P. Bertsekas, Nonlinear Programming, Athena Scientific, Belmont, Mass, USA, 1995.

[10] D. P. Bertsekas and J. N. Tsitsiklis, Parallel and Distributed Computation, Prentice-Hall, Englewood Cliffs, NJ, USA, 1989.

[11] J. F. Bonnans, J. C. Gilbert, C. Lemaréchal, and C. Sagastizábal, Optimization Numerique: Aspects Theoriques et Pratiques, Springer, Berlin, Germany, 1997.

[12] C. Lemaréchal, "Lagrangian decomposition and nonsmooth optimization: bundle algorithm, prox iteration, augmented Lagrangian," in Nonsmooth optimization: Methods and Applications, pp. 201-216, Gordon and Breach, Philadelphia, Pa, USA, 1992.

[13] Y. R. He, "Minimizing and stationary sequences of convex constrained minimization problems," Journal of Optimization Theory and Applications, vol. 111, no. 1, pp. 137-153, 2001.

[14] A. Pinkus, "Density in approximation theory," Surveys in Approximation Theory, vol. 1, pp. 1-45, 2005.

[15] A. Auslender, Optimization, Méthodes, Numériques, Masson, Paris, 1976.

[16] B. Hammer, "Universal approximation of mappings on structured objects using the folding architecture," Technical Report. Reihd P, Heft 183, Universitat Osnabruck, Fachbereich Informatic, 1996.

[17] B. Hammer and V. Sperschneider, "Neural networks can approximate mappings on structured objects," in Proceedings of the International Conference on Computational Inteliigence and Neural Network, P. P. Wang, Ed., pp. 211-214, 1997.

[18] K. Hornik, M. Stinchcombe, and H. White, "Multilayer feedforward networks are universal approximators," Neural Networks, vol. 2, no. 5, pp. 359-366, 1989.

[19] K. C. Kiwiel, "A proximal bundle method with approximate subgradient linearizations," SIAM Journal on Optimization, vol. 16, no. 4, pp. 1007-1023, 2006.

[20] J. L. Goffin, Z. Q. Luo, and Y. Ye, "Complexity analysis of an interior cutting plane method for convex feasibility problems," SIAM Journal on Optimization, vol. 6, no. 3, pp. 638-652, 1996. 


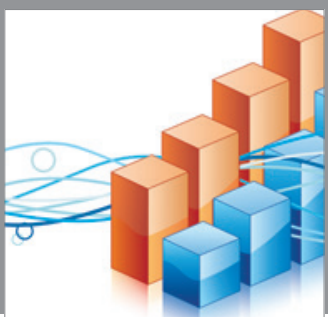

Advances in

Operations Research

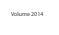

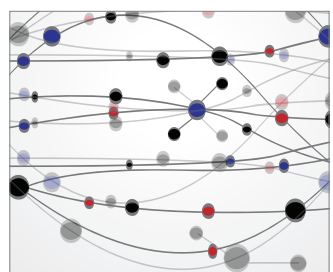

\section{The Scientific} World Journal
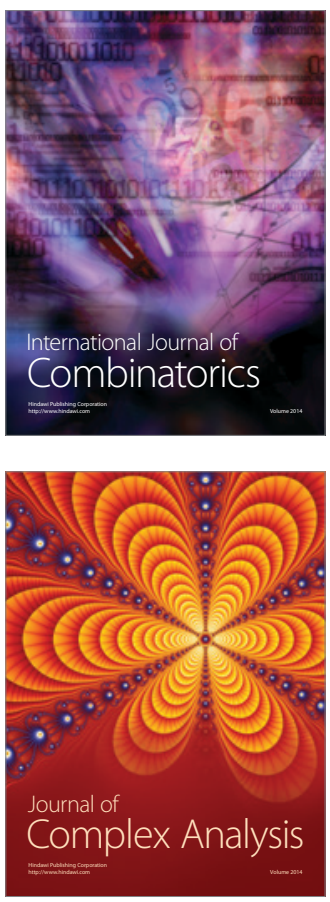

International Journal of

Mathematics and

Mathematical

Sciences
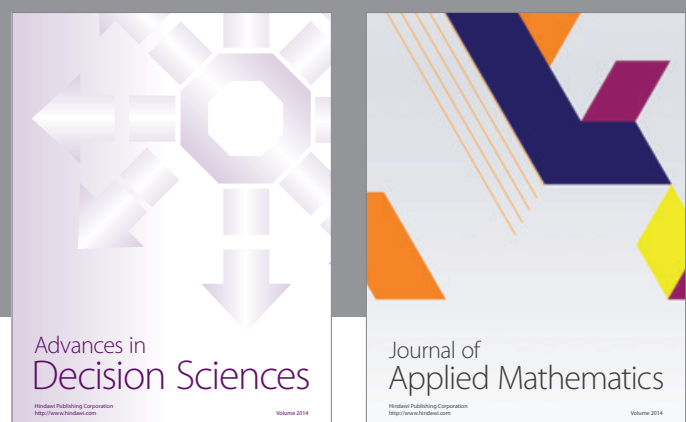

Journal of

Applied Mathematics
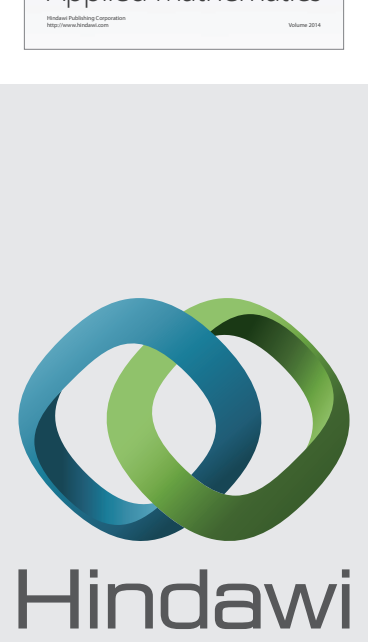

Submit your manuscripts at http://www.hindawi.com
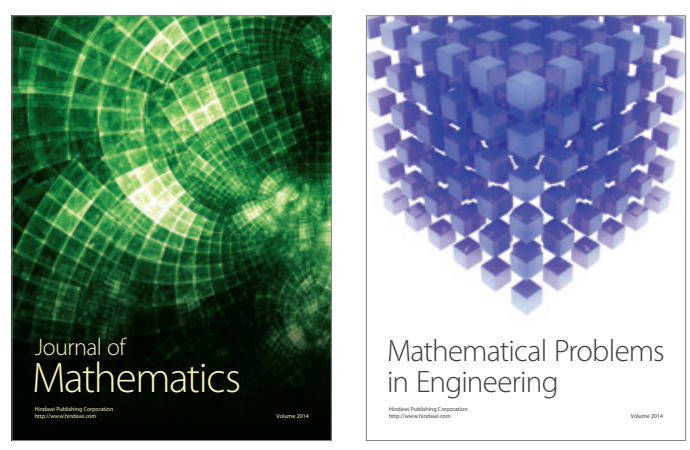

Mathematical Problems in Engineering
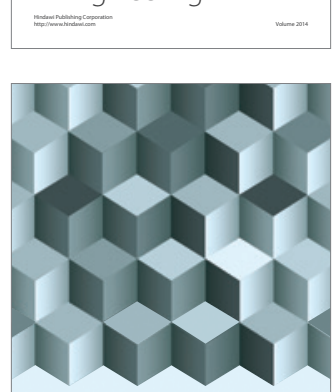

Journal of

Function Spaces
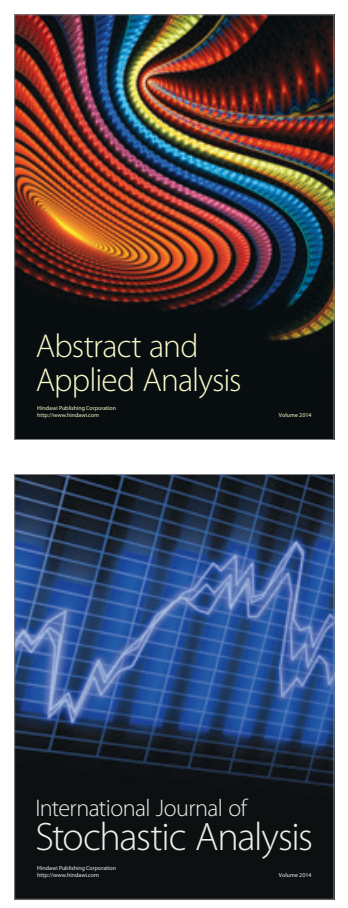

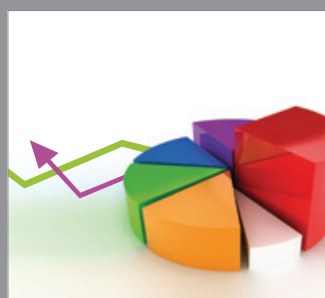

ournal of

Probability and Statistics

Promensencen
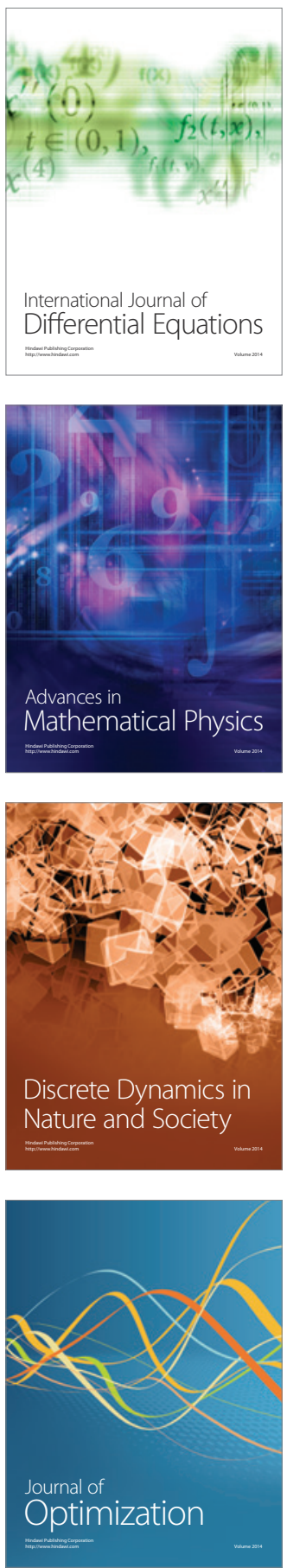